
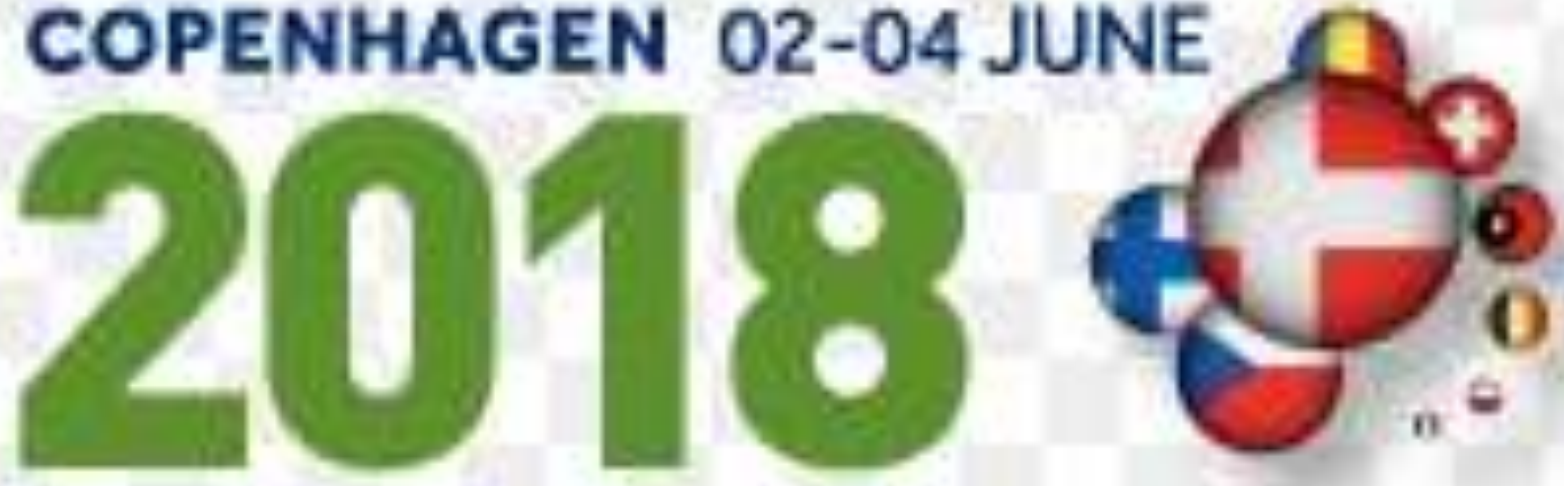

\title{
SIMULTANEOUS REGISTER WITH BIS AND STATE ENTROPY. COMPARATIVE IN THE INDUCTION WITH PROPOFOL.
}

P. Pérez Jiménez, L. Bueno Rey, P. De la Calle Elguezabal, R. Sanchez, S. Fossati Anesthesia and Critical Care department.

Hospital Clinico San Carlos, MADRID, SPAIN.

\section{BACKGROUND AND GOAL OF STUDY}

Verify the concordance and /or correlation between the values of spectral BIS and State Entropy (SE) achieved in the anesthetic induction performed with propofol.

Simultaneously determine the hypnotic depth expressed in BIS and SE values after the administration. In addition, check if this hypnotic depth is maintained for the time necessary to achieve optimal intubation conditions.

\section{MATERIALS AND METHODS}

The study population consisted of patients undergoing general or urological surgery at the Hospital Clínico San Carlos de Madrid between January 2012 and December $2013(n=47)$. The main variable refers to the anesthetic depth during induction with propofol obtained by the nondimensional analogue recording of the BIS and SE values from the beginning of the infusion of propofol until signs suggestive of excessive superficialisation were observed. Other variables that were registered where hemodynamic parameters such as $\mathrm{BP}, \mathrm{HR}$ and $\mathrm{SpO} 2$.

\section{RESULTS AND DISCUSSION}

In the comparative study, the final numbers of SE were higher than those of BIS, with a mean of 56.1 vs 49.6.

A discrepancy appeared in the analysis of the evolution of SE and $B I S$ registers since the minimum values of $S E(\min =6)$ were also lower than those of BIS $(\min =11)$ and were more distant in time, reaching earlier. As a result, the ascending slope of the state entropy was steeper than de BIS one (Figure 1)

Furthermore, it was analyzed the relationship between BIS and SE minimum values in time and in numbers. The coincidence of the minimum values of BIS and $S E$, adding $+/-10$ units as a reference, was $34,04 \%$.

Fig 2: Comparative in time

\section{CONCLUSIONS}

In those patients whose induction was performed with propofol, both BIS and SE provided a reliable guide to assess the adequacy of the hypnotic depth achieved. Likewise, the degree of depth achieved with the initial bolus, according to the records, is maintained long enough for adequate endotracheal intubation conditions. However, SE can be a parameter that provides greater safety since it ascends earlier than BIS. Besides, it has shown to be more sensitive to variations in hypnotic depth and better predictor than BIS in the anesthetic stage emergence. It may be less reliable in assessing the exact degree of hypnotic depth when the level of consciousness is very low. Both BIS and SE should be considered useful parameters to predict the patient's tendency of hypnotic depth. On the contrary, they should not be useful to acknowledge the exact depth degree that reached in every moment.

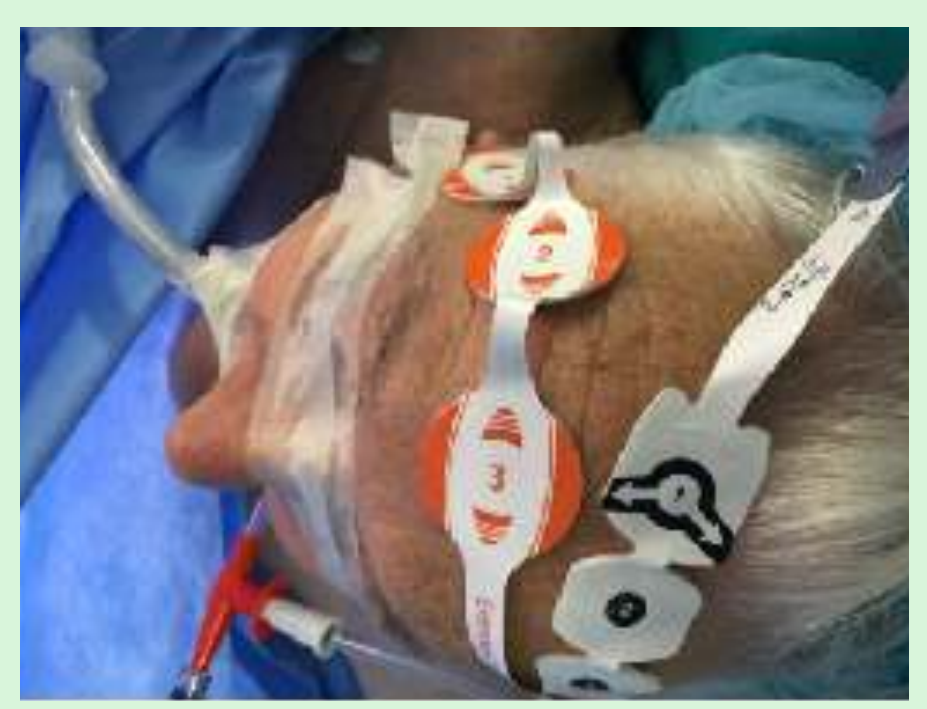

